\title{
IS THERMAL LENS SPECTROMETRY AN ABSOLUTE METHOD FOR FLUORESCENCE QUANTUM YIELD AND ABSORBANCE MEASUREMENTS
}

\author{
A. CHARTIER and J. GEORGES \\ Laboratoire des Sciences Analytiques, Bât. 308, Université Claude Bernard-Lyon I, \\ F-69622 Villeurbanne cedex, France
}

\begin{abstract}
Some anomalies observed in pulsed-laser thermal lens spectrophotometry are presented. Deviations from the expected thermal lens signal were obtained with fluorescent as well as non-fluorescent compounds. Also, transient absorption of the probe beam from long-lived triplet states may induce errors in the measurement of the true thermal lens signal.
\end{abstract}

\section{Introduction}

Thermal lens spectrophotometry (tis) is based upon non-radiative relaxation from an excited state solute. When a sample is irradiated by a laser beam, with TEMOO mode, thermal relaxation of the excited molecules dissipates heat to the solution and creates a temperature gradient. This temperature gradient generates a refractive index gradient which acts as an effective optical lens. The thermal lens is detected by its effect on the propagation of a probe laser beam, that is, by measuring the diminution in intensity of the probe laser passing through the sample on the same axis as the excitation beam.

The thermal lens signal is proportional to the focal length of the thermal lens. For a dual-beam arrangement under optimum beam modematched conditions, assuming that both beams have the same size and expand at the same rate, the signal may be expressed by the following equations:

$$
S_{\max (t=\infty)}=2.3 A P(\mathrm{dn} / \mathrm{dT}) /(1.91 \lambda \mathrm{k})
$$

with cw excitation [1], and

$$
S_{\max (t=0)}=2.3 \mathrm{~A} 3^{3 / 2} \mathrm{H}(\mathrm{dn} / \mathrm{dT}) /\left(\lambda \omega^{2} \rho \mathrm{C}_{\mathrm{p}}\right)
$$

with pulsed excitation [2], where $A$ is the decadic absorbance, $P$ the $\mathrm{Cw}$ power, $\mathrm{H}$ the pulse energy, $\lambda$ the wavelength of the probe laser, $\mathrm{d} n / \mathrm{dT}$ the refractive index gradient, $\omega$ the radius of the excitation beam into the sample and $\rho, C_{p}$ and $k$ are the density, the specific heat and the thermal conductivity of the solvent, respectively.

The tls method has been demonstrated to be a powerful technique in analytical chemistry for the detection of small absorbances. It has also been used successfully for the measurement of fluorescence 
quantum yields, for the investigation of triplet formation and triplet lifetimes and for the study of photochemical or photosensitized reactions (quantum yield, rate and enthalpy). The method may allow the direct observation of radiationless deactivation processes and the measurement of one process is based on the principle of energy conservation. The energy absorbed by a sample can be dissipated as thermal energy, radiant energy (i.e., fluorescence), or chemical energy (i.e., photochemical reactions). The energy absorbed, $P_{a}$, must be equal to the sum of the fluorescence energy, $P_{f}$, the thermal energy degraded to heat, $P_{t h}$, and the energy involved in photochemical reactions due to the enthalpy difference $\Delta H$ between the reactant and the product [3].

$$
P_{a}=2.3 \mathrm{AP}_{\mathrm{O}}
$$

where $P_{O}$ is the laser energy incident on the sample;

$$
P_{f}=2.3 \mathrm{AP}_{\mathrm{O}} Q_{\mathrm{f}}\left(\bar{\nu}_{\mathrm{f}} / \nu_{\mathrm{a}}\right)
$$

where $Q_{f}$ is the fluorescence quantum yield, and $\bar{\nu}_{f} / \nu_{a}$, the ratio of the average fluorescence frequency to the absorption frequency. The latter takes into account the stokes shift causing some deposition of heat in the sample, even when $Q_{f}=1$.

$$
P_{\text {ph }}=2.3 \mathrm{AP}_{\mathrm{O}}\left(1 / \mathrm{h} \nu_{\mathrm{a}}\right) Q_{\mathrm{ph}} \Delta \mathrm{H}
$$

where $Q_{\mathrm{ph}}$ is the quantum $y$ ield of the photochemical reaction, i.e. the ratio of the number of molecules involved in the photochemical process to the number of excited molecules. The energy released to heat is then equal to:

$$
\mathrm{P}_{\text {th }}=2.3 \operatorname{AP}_{\mathrm{O}}\left\{1-\mathrm{Q}_{\mathrm{f}}\left(\nu_{\mathrm{f}} / \nu_{\mathrm{a}}\right)-\mathrm{Qph}_{\mathrm{ph}}\left(1 / \mathrm{h} \nu_{\mathrm{a}}\right) \Delta \mathrm{H}\right\}
$$

If a sample that is optically excited can decay only by fluorescence or by heat generation, the measurement of the energy absorbed $\mathrm{P}_{a}$ and of the thermal energy $P_{t h}$ provides the fluorescence quantum yield for a simple two-level system.

In order to obtain quantitavely absorptivity or fluorescence quantum yield, the absolute energy released as heat by the excited sample should be obtained directly from the tls signal. As shown in eqs 1 and 2 , it is theoretically possible to relate the tis signal to the amount of absorbed energy that is actually dissipated as heat. However, this is very difficult because one needs to know very precisely the physical properties of the sample and a number of optical and geometrical parameters of the experimental set-up. The best way to avoid this difficulty is to compare the tls signal for an unknown sample with that of a reference absorber of known absorbance. The reference absorber is a species for which all the absorbed energy is supposed to be converted to heat in a time shorter than the time scale of the measurement.

\section{Results}

The comparison method is based on the theoretical expectation that the signal intensity should be proportional to only the thermal energy that is released from radiationless deactivation processes. Examples of incomplete recovery of the thermal lens signal for some solutes in benzene and carbon tetrachloride are reported in Table 1. 
Table 1: Qth values for various solutes in benzene and carbon tetrachloride. Time-resolved experiments with pulsed excitation. Benzophenone was the reference absorber and its $Q_{t h}$ was set to be one. From Ref.[3].

$\begin{array}{lcc} & \text { Benzene } & \mathrm{CCI}_{4} \\ \text { phthalazine } & 0.73 & 0.80 \\ \text { phenazine } & 0.72 & 0.99 \\ \text { quinoline } & 0.68 & 0.78 \\ \text { p-nitrophenol } & 0.65 & 0.63 \\ \text { o-nitrophenol } & 0.62 & 0.69 \\ \text { fluorenone } & 0.85 & 0.99\end{array}$

The triplet state of each compound was assumed to be efficiently quenched. The authors [3] have suggested that the solute dependence of the signal comes from the vibrational-mode dependence of the vibration to translation energy-transfer rate. The dissipation of the excess of vibrational energy of the solute into the solvent would depend on the solute-solvent interactions and on the frequency of the vibrational modes.

other results have been obtained for some dyes which are fluorescent or exhibit high-triplet state quantum yields (Table 2).

Table 2: Comparison of theoretical and experimental Qth values for some solutes in water. Time-resolved experiments $w i t h$ pulsed excitation. $\mathrm{CoSO}_{4}$ is the reference absorber. Fluorescein is compared with quenched fluorescein ( $\mathrm{KI}=1.35 \mathrm{M})[4]$.

$\begin{array}{lllll}\text { solute } & \text { QISC } & \text { Qf } & \text { theor. } & \text { exper } \\ & - & - & - & 1 \\ \text { Coso } & & & \\ \text { Eosin } / \mathrm{OH}^{-} & 0.76 & 0.22 & 0.80 & 0.11 \\ \text { Fluorescein } & 0.03 & 0.92 & 0.10 & 0.11 \\ \text { Fluo + KI } & - & 0 & 1 & 0.65 \\ \text { Erythrosin } & 1.07 & 0.02 & 0.98 & 0.20\end{array}$

These results show also a solute dependence of the thermal lens signal. They mean that the thermal lens method is not always accurate for measuring fluorescence quantum yields. The results of quenched fluorescein are not consistent with an other result reported previously [5] and obtained with a $\mathrm{CW}$ laser-excitation arrangement. This discrepancy may originate from the different time scales for thermal lens formation depending on the operating mode of sample irradiation ( $\mathrm{CW}$ or pulsed).

Abnormal signals may be obtained when an absorbing compound exhibits a high intersystem-crossing quantum yield, and when the triplet state is able to absorb at the wavelength of the probe laser (Figure 1). We report here the case of erythrosin whose possible photophysical processes are described as follows [6]:

\author{
absorption \\ intersystem crossing \\ triplet relaxation \\ energy transfer \\ singlet oxygen relaxation
}

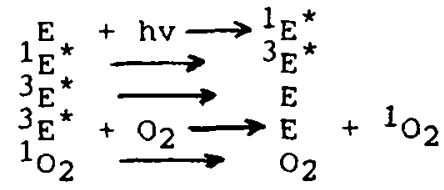




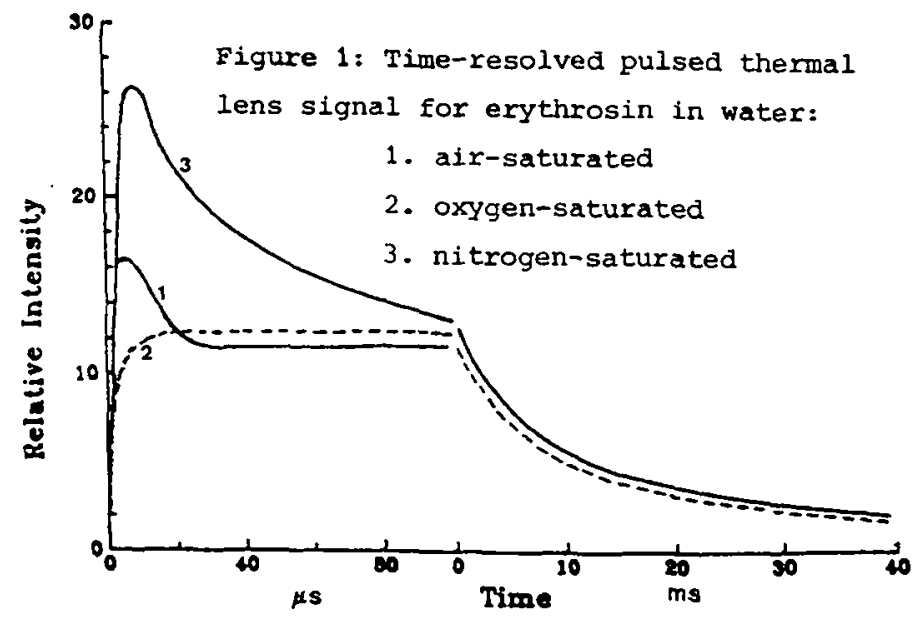

In some conditions, a signal of different nature is superimposed to the true tIs signal [6]. The superimposed signal is due to an attenuation of the probe laser intensity following its absorption by the erythrosin triplet during its lifetime. It rises up on the same time scale than that due to heat generation but decreases much faster. Depending on whether the solution is oxygen-free or oxygen-saturated, the time-resolved signal exhibits a different shape. In air-saturated solution, the superimposed signal is attributed to a residual absorption of the erythrosin triplet state which is not completely quenched by oxygen. The observed lifetime corresponds to the loss of the triplet state reacting with $\mathrm{O}_{2}$ and is related to the lifetime of $\mathrm{I}_{2}(2-3 \mu \mathrm{s})$. In oxygen-free solution, the magnitude and the lifetime of the superimposed signal are much greater. ${ }^{*}{ }^{*}$ is not quenched by oxygen and its loss is mainly governed by the intrinsic triplet lifetime (about $50 \mu \mathrm{s}$ ). In oxygen-saturated solution, $\mathrm{B}^{\star} \mathrm{s}^{\mathrm{s}}$ completely quenched by $\mathrm{O}_{2}$ so that the transient absorption signal is suppressed. Errors in the valuation of the thermal energy may originate from this superimposed signal. In pulsed-laser thermal Iens spectrometry, the maximum signal expressed by eq. 2 has roughly a constant value on the time scale of several tens microseconds, i.e. between a short time scale corresponding to the signal rise time ( $\mu$ s) and a more longer time scale (ms) corresponding to the decay of the thermal lens signal. Thus, the true signal may be over-estimated if the signal is sampled with a delay lower than $30 \mu s$ in air-saturated solution, or lower than $200 \mathrm{\mu s}$ if the solution is deoxygenated.

\section{References}

[1] Harris J.M., in Analytical Applications of Lasers, Piepmeier E.H. (ed.), wiley-Interscience, New York, 1986, Vol. 87, p. 451 .

[2] Mori K., Imasaka T. and Ishibashi N., Anal. Chem. 54 (1982) 2034 .

[3] Terazima M., Horiguchi M. and Azumi T., Anal. Chem. 61 (1989) 883 .

[4] Chartier A., Georges J. and Mermet J.M., Chem. Phys. Letters 171 (1990) 347 .

[5] Shen J. and Snook R.D., Chem. Phys. Letters 155 (1989) 583.

[6] Chartier A., Georges J. and Mermet J.M., Spectrochim. Acta 46A (1990) 1737 . 